\author{
Volkmar A. Wirth \\ Marcus Schuermann
}

\title{
Hyperthermie - eine innovative Therapieoption der Integrativen Onkologie
}

Die Hyperthermie gewinnt in den letzten Jahren mehr und mehr Befürworter, weil sie eine nichtoperative, besonders schonende, praktisch nebenwirkungsfreie, aber dennoch sehr effektive Therapie ist. Sie verstärkt die Wirkungen von onkologischen Standardmethoden (Strahlen- und Chemotherapie) und lässt sich optimal mit diesen kombinieren. Die Ergebnisse experimenteller und klinischer Studien der jüngeren Zeit weisen zudem darauf hin, dass neben direkten zellschädigenden thermischen Effekten sowohl eine Hemmung der Angiogenese als auch eine Förderung der Gewebeperfusion, Gewebeoxigenierung und Immunsurveillance eine Rolle spielen. Hyperthermie ist somit eine innovative Behandlungssäule in einem multimodalen, integrativen und damit ganzheitlichen Therapiekonzept.

Mit der Entwicklung und Einführung der Radiotherapie und später der Chemotherapie in den 50er-Jahren wurde die in der ersten Hälfte des 20. Jahrhunderts vielversprechende medizinische Hyperthermieforschung zunächst fast vollständig unterbrochen. Damit teilte die Hyperthermie das Schicksal vieler physikalischer Heilverfahren, die in der Dominanz modernerer, industriell getragener Verfahren zunächst vernachlässigt wurden.

Das wissenschaftliche Interesse hat sich dann in den 70er-Jahren erneut gesteigert: einerseits mit der Entwicklung nichtinvasiver elektromagnetischer Einstrahlung mittels Radiooder Mikrowellen, andererseits mit der Entwicklung multimodaler Therapiekonzepte in Verbindung mit Radiotherapie und Chemotherapie [2].

Heute existieren umfangreiche Erfahrungen zum kombinierten Einsatz der Hyperthermie bei vielen Krebsarten. Insbesondere zur lokoregionalen Therapie liegen zwischenzeitlich zahlreiche kontrollierte Studien vor, die die Wirksamkeit im Allgemeinen belegen, während auch die tumorbezogenen Indikationen und Limitationen weitgehend bekannt sind.

\section{Formen der Hyperthermie}

Die Hyperthermie wird grundsätzlich in folgende zwei Formen eingeteilt:

- Aktive Hyperthermie: Vom Körper selbst erzeugtes Fieber mittels intravenöser Gabe von Pyrogenen.

- Passive Hyperthermie: Erhöhung der Temperatur im gesamten Körper bzw. in Körperteilbereichen durch Geräteeinsatz von aussen.

In der passiven Hyperthermie wird zudem zwischen Ganzkörper- und lokoregionalen Hyperthermieformen unterschieden: Ganzkörperhyperthermie (GKHT) wird je nach erreichter Temperatur als moderate (bis $38,5^{\circ} \mathrm{C}$ ), intermediäre $\left(39-41,5^{\circ} \mathrm{C}\right)$ und extreme GKHT $\left(41,5-42,8^{\circ} \mathrm{C}\right)$ bezeichnet. Bei der lokoregionalen Hyperthermie (LRHT) wird eine begrenzte Körperregion oder ein Organ übererwärmt. 
Von der Hyperthermie im engeren Sinn abzugrenzen ist die Thermotherapie, bei der Gewebe mit Temperaturen oberhalb $45{ }^{\circ} \mathrm{C}$ direkt mit Laser oder hochfokussiertem Ultraschall zerstört wird, z.B. die Laser-induzierte Thermotherapie (LITT) oder «highly focussed ultrasound» (HIFU). Daneben existieren Sonderformen wie die hypertherme Extremitätenperfusion oder die im operativen Verfahren angewandte peritoneale Hyperthermie, deren Einsatz und Weiterentwicklung derzeit in akademischen Zentren erfolgt [3].

\section{Formen der lokoregionalen Hyperthermie}

Die LRHT kommt bei lokal begrenzten Tumoren sowohl an der Oberfläche als auch in der Tiefe des Körpers zum Einsatz. Zur Energieübertragung und lokalen Wärmeerzeugung wird einer von drei physikalischen Effekten angewandt (Abb. 1):

- Magnetfeld (induktive Kopplung);

- elektrisches Feld (kapazitive Kopplung);

- elektromagnetische Strahlung (Strahlungskopplung oder Antennensystem).

Dank seiner selektiven und gezielten Wirkung ist besonders die kapazitive Kopplung zukunftweisend. Bei dieser Technik der LRHT erzeugen zwei Metall-Elektroden ein elektrisches Wechselfeld. Der Körper des Patienten wirkt hierbei ähnlich wie in einem Kondensator als Isolierstoff (Dielektrikum). Zwischen den zwei Elektroden kommt ein elektrisches Feld zustande (Abb. 2).

Da die Leitfähigkeit und die Dielektrizität im Tumorgewebe signifikant grösser sind, der elektrische Widerstand dagegen geringer ist als im gesunden Gewebe, fokussiert sich über die bessere Leitfähigkeit der Energieeintrag im Tumorgewebe. Tumorzellen werden dadurch selektiver erwärmt als Normalgewebe. Dieses Prinzip der «Autofokussierung» erlaubt demnach auch die Therapie an

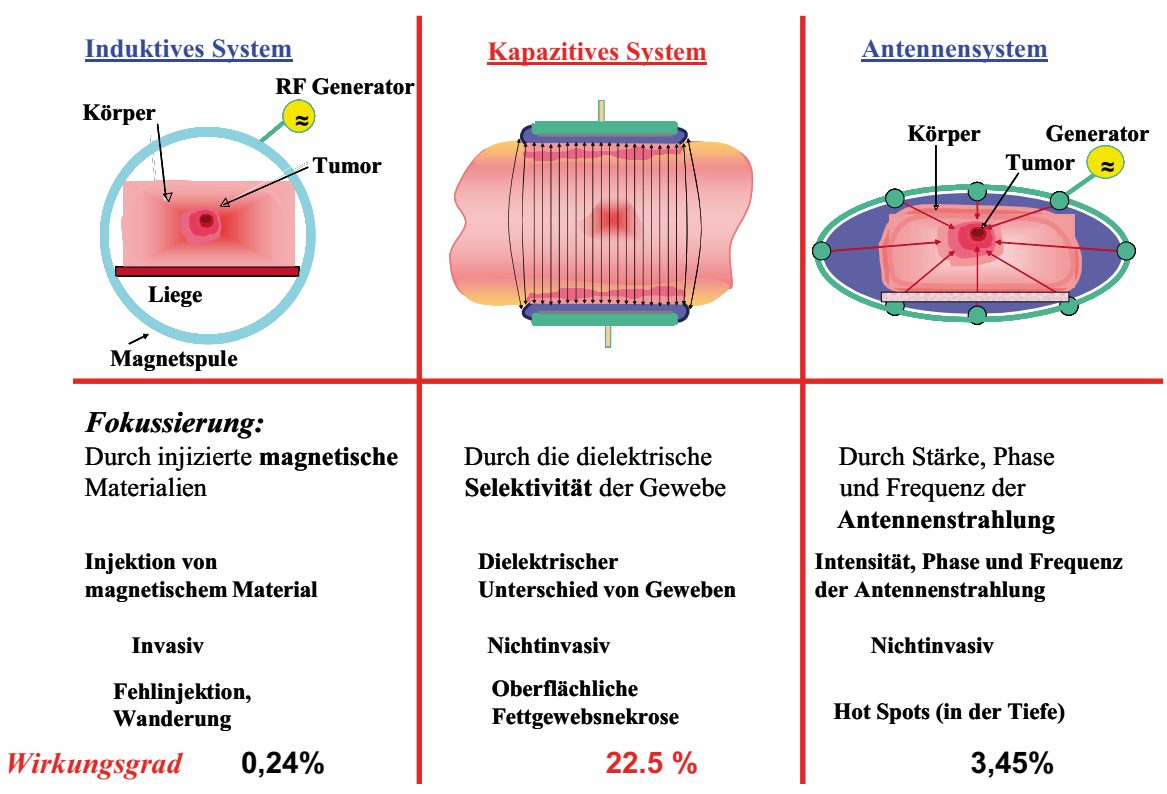

Abb. 1. Unterschiedliche Hyperthermiesysteme, Vor- und Nachteile, Wirkungsgrad (Quelle: H. Sahinbas [3]).

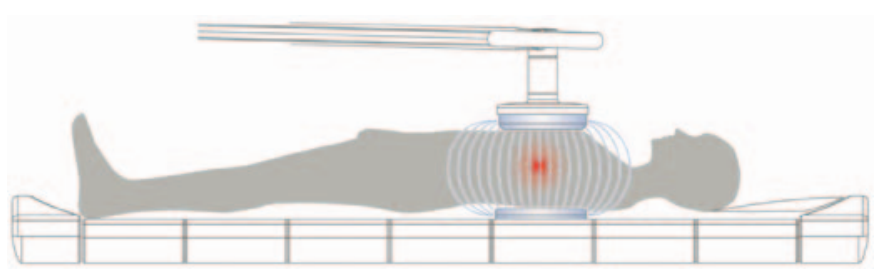

Abb. 2. Elektrodenanordnung bei der kapazitiven Hyperthermie (Abdruck mit freundlicher Genehmigung der Fa. Celsius42+ GmbH).

beweglichen Tumoren wie Lungentumoren oder im Bereich thermosensibler Organe wie Gehirn oder Auge. Das Verfahren bietet somit ein breites Indikationsspektrum.

Während Magnetfeldverfahren noch weitgehend in der klinischen Testung sind, ist die lokale Überwärmung mittels phasengesteuerter Antennensysteme im deutschsprachigen Raum vor allem in universitären Einrichtungen weit vertreten. Die Entwicklung dieser Geräte erfolgte weitgehend in den 80er- und 90er-Jahren, sodass ihre Wirkung und Anwendbarkeit am meisten während der letzten zwei Jahrzehnte untersucht werden konnte. Der Nachteil dieser Verfahren ist die inhomogenere Temperaturverteilung ("hot spots») an Grenzflächen, wie sie etwa an Knochen, Übergängen zu Luft oder fetthaltigem Gewebe entstehen. Dadurch wird z.B. der Einsatz im Thoraxbereich begrenzt.

\section{Wirkmechanismen der Hyperthermie}

Neben den auch durch Fieber hervorgerufenen systemischen Wirkungen, wie zum Beispiel die Aktivierung des Immunsystems und die Schmerzlinderung, bedingt die Hyperthermie eine Reihe weiterer Mechanismen, die jeweils direkt oder indirekt schädigend auf das Tumorgewebe einwirken: - Minderdurchblutung im Tumor durch Gefässveränderung (Abb. 3), - Azidose der karzinogenen Zellen,

- Energieverarmung im Tumorgewebe,

- Veränderung der Zellmembran von Tumorzellen,

- direkte Tumornekrose, 


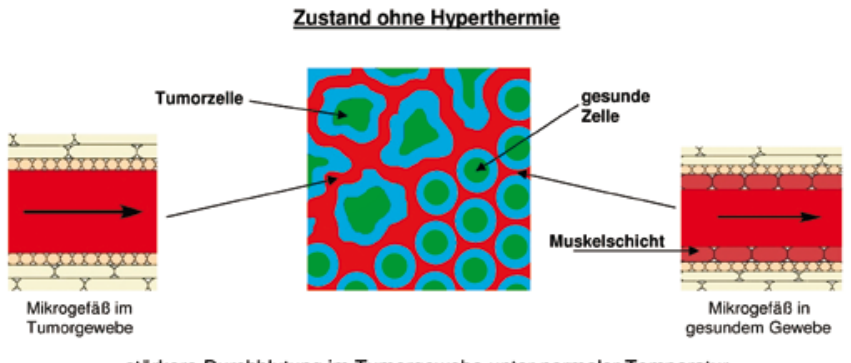

stărkere Durchblutung im Tumorgewebe unter normaler Temperatur

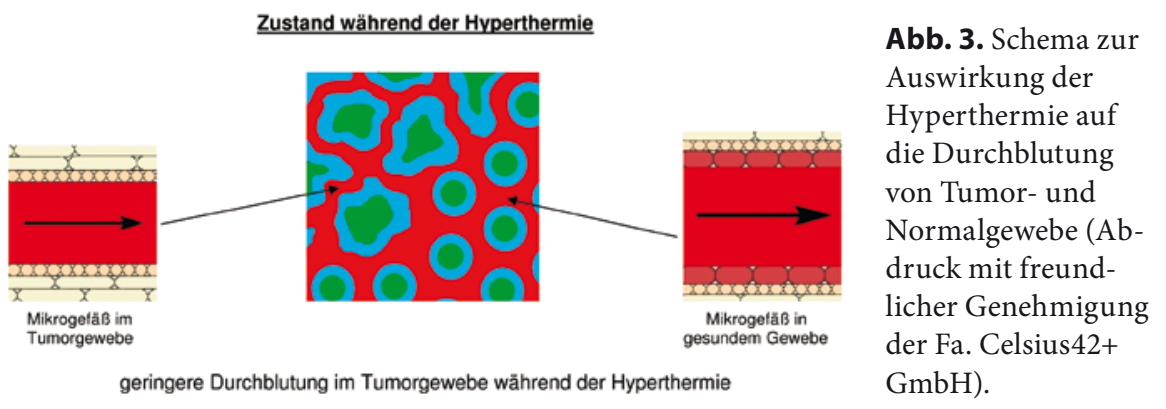

- Abnahme der DNA-Replikationsgeschwindigkeit der Tumorzellen,

- Mikrothrombosen im Tumor (angiogenetischer Block),

- Zunahme der Produktion von Hitze-Schock-Proteinen im Tumorgewebe, die als Erkennungsstruktur für das Immunsystem dienen.

\section{Studienlage zur Wirksamkeit der Hyperthermie}

Eine Reihe von existierenden klinischen Studien zum Einsatz von Hyperthermie in verschiedenen onkologischen Indikationen wurde vor kurzem von Horsman und Overgaard in Form einer Metaanalyse publiziert [4]. Dabei wurden 23 Studien mit insgesamt 1861 Patienten mit unterschiedlichen Tumorlokalisationen berücksichtigt. Der Vorteil der regionalen Tumorkontrolle unter einer Kombination von Strahlentherapie und LRHT (52\%) war gegenüber der alleinigen Strahlentherapie (38\%) hoch signifikant. Zusammenfassend ist die Hyperthermie wohl der potenteste Radiosensibilisator, der zurzeit bekannt ist. In-vivo-Studien haben ergeben, dass der Effekt der Strahlentherapie durch die Hyperthermie um den
Faktor 1,2-2,5 verstärkt wird. Man konnte zudem Tumorarten identifizieren, bei denen der zusätzliche Einsatz der Hyperthermie auch eine Verlängerung der Überlebenszeit mit Zunahme der kompletten Remissionsraten von $31 \%$ auf $67 \%$ erbrachte $[5,6]$.

Ergebnisse der Analyse führten die Autoren zu folgendem Schluss: «Die Skepsis gegenüber der Hyperthermie sollte überwunden werden, da sie klinisch effektiv ist, und das ist wahrscheinlich das wichtigste Argument, das für Krebspatienten zählt» [4].

Auf dem vorletzten Treffen der American Society of Clinical Oncology (ASCO) wurde eine prospektive Phase-II-Studie aus Deutschland [7] präsentiert, die die Wirksamkeit der LRHT bei hochmalignen Hirntumoren in fortgeschrittenen Stadien (Astrozytom/Glioblastom WHO Grad III und IV) untersucht. Die bekannte Gesamtüberlebenszeit für diese Patientengruppe beträgt lediglich circa 12 Monate. Erstaunlicherweise konnte bei Patienten, die sich zusätzlich zur Radio-/Chemotherapie einer regelmässigen LRHT unterzogen hatten, ein besseres Ansprechen mit signifikanter Verlängerung der Überlebenszeit auf 23,5 Monate beobachtet werden. Dieses positive Ergebnis wird zusätzlich durch die Tatsache unterstützt, dass 23 der 126 Patienten mit Glioblastomen (18,3\%) am Ende der 3-jährigen Beobachtungszeit am Leben geblieben waren.

\section{Bei welchen Krebsarten wird die lokoregionale Hyperthermie erfolgreich eingesetzt?}

Die bisherige wissenschaftliche Evidenz (Phase-II-Daten) sowie klinische Erfahrung lässt für diese Methode folgende Indikationen definieren (jeweils im Stadium des lokal fortgeschrittenen Wachstums) [4-6, 8, 9]:

- Gynäkologische Tumoren (Zervix-, Vaginal- und Ovarialkarzinom),

- Pankreaskarzinom,

- Urothelkarzinom der Blase,

- Prostatakarzinome,

- Rektumkarzinome,

- Peritonealkarzinose,

- Weichteiltumoren einschliesslich Mesotheliome,

- Tumoren im Bereich der Brustwand (Mammakarzinom),

- Hals-Nasen-Ohren-Tumoren (insbesondere solitäre Lymphknotenmetastasen),

- Hirntumoren (Glioblastom, Astrozytom) und Hirnmetastasen.

Weniger dokumentiert und daher noch umstritten ist derzeit die Thermotherapie von:

- Lymphknotenmetastasen und örtlich begrenzten Lymphomen,

- Tumoren des Gastrointestinaltrakts (Magen- und Kolonkarzinom),

- primären oder metastatischen Tumoren im Bereich der Lunge, des Mediastinums und der Leber.

Im Gegensatz zu Operation, Chemotherapie und Strahlentherapie ist die Hyperthermie nahezu nebenwirkungsfrei. Nach der Therapie kann es $\mathrm{zu}$ einer gewissen Müdigkeit und in seltenen Fällen zu leichter Temperaturerhöhung kommen. Letztere wird als Ausdruck der vermehrten Nekrose maligner Zellen mit entsprechender 
Aktivierung des Immunsystems erklärt. Selten kommt es zu lokaler Überhitzung etwa im subkutanen Fettgewebe, extrem selten zu Fettgewebsnekrosen.

Bekannte Kontraindikationen für die LRHT-Behandlung sind Herzschrittmacher und andere Geräte, die durch Einwirkung elektromagnetischer Felder geschädigt werden könnten. Implantierte Stents stellen je nachdem eine relative Kontraindikation dar, inwieweit eingewebte Metalleinlagen selbst als Antenne den Eintrag von Mikro-/Radiowellen fokussieren können. Im Einzelfall kann hier der Hersteller kontaktiert werden. Im ungewissen Fall ist auf die Therapie zu verzichten; andernfalls kann das Stufenprofil der Behandlung flacher gewählt werden, und es sollte speziell auf überwärmungsbedingten Lokalschmerz geachtet werden.

\section{Lokoregionale Tiefenhyperthermie in der Aeskulap-Klinik}

Seit März 2009 verfügt die AeskulapKlinik in Brunnen über ein modernes, effizientes Gerät für kapazitive LRHT (Thermo Cancer Select der Fa. Celsius42+ GmbH) (Abb. 4). LRHT wird indikationsbezogen sowohl stationären als auch ambulanten Patienten als wirkungsverstärkende Therapie zur laufenden onkologischen Standardtherapie angeboten. Nach einer eingehenden Evaluation jedes Patienten wird die LRHT in der Regel 2-mal/ Woche für jeweils $1 \mathrm{~h}$ eingesetzt. Eine erste Phase umfasst jeweils $10-12$ Behandlungen; danach kann dieser Rhythmus weitergeführt werden. Seltener kann dies auch als Fortsetzung im Sinne einer Erhaltungstherapie (ca. alle 1-2 Wochen) über längere Zeit erfolgen. Der Energieeintrag erfolgt in Stufen von jeweils $10 \mathrm{~min}$, beginnend mit einem flachen Profil bis maximal 60 Watt in der ersten Sitzung und danach rasch steigend bis 100 Watt bis zur jeweils individuell auszutestenden
Maximalstufe von circa 150 Watt Eintrag, erreichbar nach etwa 8-10 Sitzungen.

Die bisherige Erfahrung in mehr als 600 Sitzungen zeigt, dass die LRHT eine ausgesprochen gute Toleranz aufweist, wobei es in wenigen Einzelfällen aber auch zu lokalen schmerzhaften thermischen Reaktionen kommen kann (bislang 6 Fälle von 600 Sitzungen). Problemzonen sind hier der ventrale Rippenbogen wie auch mesenteriale Tiefenregionen. In einem Fall konnte eine subkutane Gewebeeinschmelzung mit Fibrosierungsreaktion sonografisch dokumentiert werden. Hautrötungen im Applikationsgebiet wurden nur vereinzelt beobachtet, in einem Fall auch eine Verbrennung II. Grades. Zum Wirkungsgrad sind derzeit abgesehen von Einzelfällen noch keine Aussagen möglich.

\section{Schlussfolgerung}

Zusammenfassend muss die LRHT als ein derzeit die konventionelle Behandlung verstärkendes Verfahren in der

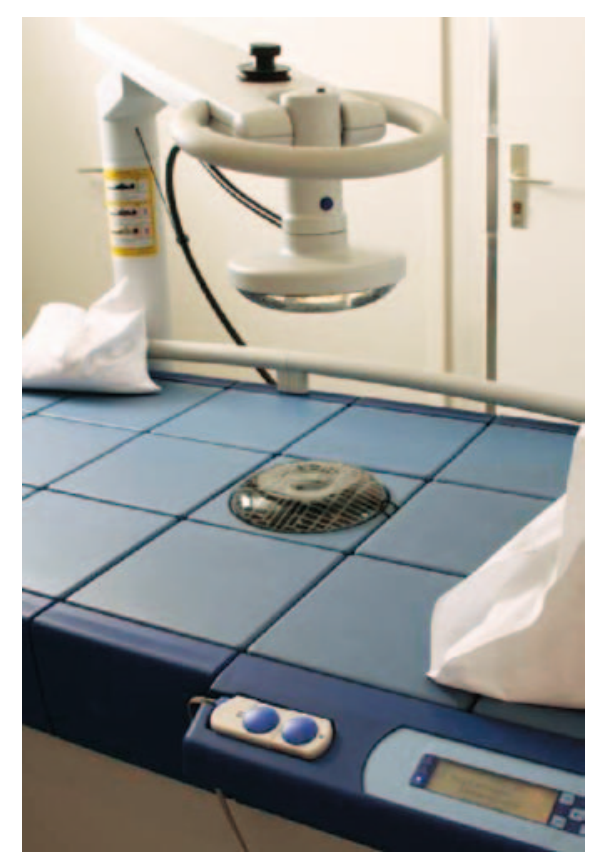

Abb. 4. Celsius TCS Thermo-CancerSelect-Gerät für die lokoregionale Tiefenhyperthermie an der Aeskulap-Klinik.
Onkologie betrachtet werden. Sie kann als Kombinationsverfahren oder in Ausnahmefällen als Monotherapie bei diversen fortgeschrittenen, zum Teil inoperablen Tumoren oder deren Metastasen eingesetzt werden. Die Kombination der LRHT mit anderen Standardmassnahmen (insbesondere mit Radio- wie auch mit Chemotherapie) führt zur Verlängerung der medianen Gesamtüberlebenszeit und ist zwischenzeitlich gut in zahlreichen PhaseII-Studien dokumentiert. Die angewandte LRTH-Behandlung wird in der Regel von den Patienten sehr gut toleriert.

\section{Literatur}

1 Baronzio GF, Hager ED: Hyperthermia in Cancer Treatment: a Primer. New York, Springer/ Landes Bioscience, 2006.

2 Adamietz IA, Wust P: Hyperthermie in der nichtkonventionellen Therapie. Der Onkologe 2004;10:276-283.

3 Sahinbas H: Hyperthermie in der modernen medizinischen Behandlung, Sonderdruck, 2008. www.dght-ev.de/fileadmin/downloads/Hyperthermie_Formen_Sahinbas.pdf.

4 Horsman MR, Overgaard J: Hyperthermia: a potent enhancer of radiotherapy. Clin Oncol (R Coll Radiol) 2007;19:418-426.

5 Overgaard J: The current and potential role of hyperthermia in radiotherapy. Int J Rad Biol Physics 1989;16:535-549.

-6 Van der Zee J, Gonzalez D, van Rhoon GC, van Dijk JDP, Putten WLJ, Hart AAM: Comparison of radiotherapy alone with radiotherapy plus hyperthermia in locally advanced pelvic tumours: a prospective, randomized, multicentre trial. Lancet 2000;355:1119-1125.

7 Hager ED, Sahinbas H, Groenemeyer DH, Migeod F: Prospective phase II trial for recurrent high-grade malignant gliomas with capacitive coupled low radiofrequency (LRF) deep hyperthermia. ASCO 2008; \#2047.

8 Maluta S, Pioli F, Dall'Oglio S, Romano M Impact of hyperthermia combined with radiochemotherapy in locally advanced pancreatic cancer. 10th International Congress On Hyperthermic Oncology (ICHO). München, 2008(abstract).

9 Tschoep K, Boeck S, Berger F, Maier V, AbdelRahman S, Kuhlencordt M, Salat C, Schmidt M, Heinemann V, Issels R: Gemcitabine (GEM) + Cisplatin (CIS) combined with regional hyperthermia (RHT) in patients with GEM-pretreated pancreatic cancer: results of the ESHO phase II trial. 10th International Congress On Hyperthermic Oncology (ICHO). München, 2008(abstract). 